\title{
The response criterion, the stimulus configuration, and the relationship between brightness contrast and brightness constancy*
}

\author{
BARRY R. HAIMSON \\ Southeasterm Massachusetts University, North Dartmouth, Massachusetts 02747
}

\begin{abstract}
Four experiments were designed to investigate the nature of the relationship between brightness contrast and brightness constancy while controlling the response criterion, the area of the surround, the stimulus configuration, and the mode of appearance of the modulus target. Ten Os in each of the four experiments estimated the apparent whiteness or brightness of targets with different contrast ratios. All targets were viewed at several illumination levels. Most constancy (whiteness and brightness) functions displayed shallow slopes that reflected a good approximation to constancy. The functions within Experiments I, III, and IV were vertically displaced and parallel; those in Experiment II were vertically displaced and increased in slope. This suggests that decreasing the contrast ratio had no effect on the tendency towards constancy when the area of the surround was greater than that of the target but resulted in a decrease in constancy when the area of the surround was equal to that of the target.
\end{abstract}

Since the initial findings suggesting a coexistence of the conditions necessary for brightness constancy and contrast (Katz, 1935; Gelb, 1929; Henneman, 1935), there have been a number of investigations aimed at demonstrating a systematic relationship between the two sets of phenomena. Some investigators (Leibowitz, Myers, \& Chinetti, 1955; Kozaki, 1963) used targets with clear surface quality, while others (Hess \& Pretori, 1894; Jameson \& Hurvich, 1961; Wallach, 1948) used projected or transilluminated targets that came very close to surface character. Moreover, many experimental demonstrations of brightness contrast (Heinemann, 1955; Horeman, $1963,1965)$ have produced configurations which have been viewed as comparable to those in a constancy situation. Since certain experimental outcomes were predicted if constancy prevailed, these experimental conditions enabled a direct comparison of the effects of experimental variables on both contrast and constancy.

Freeman (1967) pointed out some difficulties in the brightness contrast interpretation of brightness constancy. For example, perfect constancy was rarely achieved in any study that measured brightness (matched luminance) over illumination changes. In most instances, there was only an approximation to brightness constancy. Further, the literature contained two sets of contradictory findings concerning the relationship between the magnitude of contrast and the approximation to brightness constancy.

\footnotetext{
*This research was submitted in partial fulfillment of the requirements for the degree of Doctor of Philosophy at Boston University.
}

One group of investigators (Hess \& Pretori, 1894; Leibowitz et al, 1955; Horeman, 1965; Jameson \& Hurvich, 1961) have suggested that the slope of the function relating changes in the apparent brightness of the test figure in a simple configuration (disk-annulus or rectangle-frame) and the illumination falling on both the test and surround fields depended upon the value of the contrast ratio (luminance of center figure/luminance of surround). Accordingly, with contrast ratios less than unity, the slope of the brightness function systematically decreased until at some point a slope of 0 was obtained (i.e., brightness constancy). Moreover, Jameson and Hurvich (1961) noted that further decreases in the contrast ratio produced the paradoxical outcome of a negative slope (i.e., apparent brightness decreased as a function of increases in the overall level of illumination). On the other hand, others (Wallach, 1948; Kozaki, 1963) found that the principal effect of manipulating the contrast ratio was to displace the brightness function by a constant value, i.e., to produce a series of brightness functions that were vertically displaced and parallel.

To clarify previous conflicting reports, a series of pilot studies (Haimson, 1970) was conducted to investigate the relationship between brightness contrast and brightness constancy under conditions comparable to those of Heinemann (1955). Os viewed disk-annulus configurations with different contrast ratios $(-1 \text { to }-15 \mathrm{~dB})^{1}$ under different levels of illumination $(60$ to $100 \mathrm{~dB}){ }^{2}$ In some studies, the Os adjusted a comparison disk without an annulus located to the left of the disk-annulus configuration; in others, the disk was used as a modulus in a 
magnitude estimation procedure. The obtained matched luminance and brightness functions did not display any systematic trends.

$A$ post hoc evaluation of the experimental conditions suggested the possibility that Os experienced difficulty in using a comparison field (or modulus) at a particular level of brightness as a criterion for the brightness of a test field viewed in the surface mode. That is, under these experimental conditions, the test field varied on the whiteness dimension from black to white while the modulus varied on the brightness dimension from dim to bright.

Previous investigations have also been concerned with the distinction between brightness and whiteness. Katz (1935) equated whiteness with an O's response to the object's surface color; brightness, to its insistence (total amount of reflected or transmitted light). Henneman (1935) uncovered evidence for individual differences in the O's observation attitude. Those adopting an object-directed attitude could separate reflectance from total luminance and make a reflectance match (a constancy response); those adopting a photographic attitude responded to the total luminance (brightness) and made a luminance (nonconstancy) match. In a later study, Henneman (1935) manipulated the observation attitude through appropriate instructions and found that it was possible to shift Os from one attitude to another. Landauer and Rodger (1964) reported similar findings.

It is possible that some of the contradictory evidence concerning the relationship between brightness contrast and constancy resulted from ambiguous instructions which failed to specify clearly the desired response dimension (whiteness or brightness). Moreover, previous studies have utilized a variety of stimulus configurations. It is plausible, therefore, that there may have been some interaction between the stimulus configuration and the relationship between brightness contrast and constancy.

The present series of experiments were designed to evaluate the relationship between brightness contrast and perceptual constancy (whiteness or brightness) while controlling the response criterion and stimulus configuration. In Experiments I and II, the modulus was viewed in the surface mode and the Os were instructed to adopt a whiteness criterion. The two experiments differed in terms of the size of the annulus surrounding the test stimulus. A previous investigation by Stevens (1967) revealed that the inhibitory effect of an inducing field depended upon its size. By comparing the two experiments, one can assess the influence of the size of the surround and contrast ratio on the tendency towards whiteness constancy. The test stimulus configurations used in Experiment III were the same as those in Experiment I. However, the modulus appeared in the aperture mode and the Os were instructed to adopt a brightness criterion. By comparing Experiments I and III, one can evaluate the influence of the response criterion. Experiment IV was designed to replicate the findings of Jameson and Hurvich (1961). Os were instructed to adopt a whiteness criterion and were presented a modulus and stimulus configuration comparable to those used by Jameson and Hurvich (1961).

\section{GENERAL METHOD}

The experimental apparatus and procedure were basically similar in all experiments. Departures from the basic apparatus and procedure will be explained within the description of each experiment.

\section{Apparatus}

Test stimulus patterns, differing in contrast ratio and consisting of a test field and a surround, appeared at a distance of $76.2 \mathrm{~cm}$ in the O's frontoparallel plane. They were composed of Wratten No. 96 neutral density filters mounted between flashed opal and clear glass plates. The entire configuration was transilluminated by a $500-W$ projector located in a room adjoining O's station. The beam from the projector passed in turn through a mechanical shutter, heat absorbing glass, an aperture with a diameter of $5.08 \mathrm{~cm}$, a Wratten No. 96 neutral density filter, and the test stimulus pattern, which was centered in an opening in the wall separating the $O$ 's station from the projection equipment. The area surrounding the opening was sprayed a flat black. The only thing visible to the $\boldsymbol{O}$ was the test stimulus configuration. Variation in the filter density produced four levels of illumination. All luminance measurements were made with a Macbeth illuminometer.

\section{Subjects}

Ten experimentally naive adults served as Os in each of the four experiments.

\section{Procedure}

A magnitude estimation procedure was used to determine the manner in which apparent whiteness or brightness varied as a function of changes in the level of illumination falling upon the test stimulus pattern. At the beginning of the session, $E$ gave $O$ instructions concerning the response dimension, pointed to the modulus, and told $O$ to call it 100 . With this as a reference value, $O$ was instructed to assign to each test stimulus a number proportional to its whiteness (or brightness). Each estimation was given after two 1 -sec exposures of the test stimulus configuration under free-viewing binocular observation conditions. After a 10-min adaptation to the reference configuration, each $O$ made two estimates of the whiteness (or brightness) of each of the contrast ratios under the four levels of illumination. Each combination of illumination level and contrast ratio was presented randomly. The reference configuration was present during the entire session, and $O$ could refer to it at any time.

\section{EXPERIMENTS I AND II}

These experiments were designed to evaluate the influence of the area of the annulus surrounding the test field on the tendency towards whiteness constancy with instructions to adopt a whiteness criterion. Further, it was thought that the reference stimulus (an illuminated square) used in Experiment I interfered with the whiteness estimates given to transilluminated disks. Therefore, the reference 
stimulus in Experiment II was changed to a transilluminated disk-annulus configuration equivalent in size to the test stimulus configuration.

\section{Method}

In both experiments there were six test stimulus patterns, consisting of a disk (visual angle $=1 \mathrm{deg}$ ) surrounded by an annulus (visual angle $=2.6 \mathrm{deg}$ in Experiment $\mathrm{I}$; visual angle $=$ $1.4 \mathrm{deg}$ in Experiment II). In Experiment I, the ratio of the area of the annulus to the area of the disk was $6: 1$; in Experiment II, the ratio was $1: 1$. Each test stimulus pattern had a different contrast ratio: $-1,-3,-6,-9,-12$, and $-15 \mathrm{~dB}^{1}$ and was viewed under four levels of illumination: $70,80,90$, and $100 \mathrm{~dB}^{2}$ Under these conditions, the luminance of the annulus equaled the illumination level; the disk luminance equaled the annulus luminance plus the contrast ratio (i.e., the luminance of a disk with a $-3-d B$ contrast ratio and viewed against a $70-\mathrm{dB}$ annulus was equivalent to $67 \mathrm{~dB}$ ).

In Experiment I, the reference stimulus (modulus) was a gray square, reflectance $(R)=17.4 \%$, which subtended a visual angle of 2 deg. It appeared as a member of a series of gray squares placed on the wall in the O's station $45.5 \mathrm{~cm}$ above the opening in which the test stimulus pattern appeared. The entire gray series was illuminated by a $500-W$ projector located at the rear of the O's station. The luminance of the whitest square $(R=84.9 \%)$ was $64 \mathrm{~mL}(88 \mathrm{~dB})$; the luminance of the background was $75.7 \mathrm{~dB}$. Under these conditions, the squares appeared in the surface mode. The reference configuration and the reflectance values of all squares are shown in Fig. 1.

In Experiment II, the modulus was composed of a Wratten No. 96 neutral density filter mounted between flashed opal and clear glass plates. It subtended a visual angle of $1.4 \mathrm{deg}$. The modulus-background was an annulus whose area bore a $1: 1$ ratio with the area of the modulus. The configuration (modulus and modulus background) appeared at a distance of $76.2 \mathrm{~cm}$ to the left of the $O$ and was transilluminated by a series of incandescent lamps. Throughout the experiment, the modulus background was held constant at $64 \mathrm{~mL}(88 \mathrm{~dB})$ while the modulus was constant at $81 \mathrm{~dB}$.

In both experiments, the Os were given instructions concerning the whiteness dimension. In Experiment $I, E$ pointed to the squares in the reference stimulus configuration with the highest, lowest, and middle reflectance values, and informed $O$ that the one with the highest reflectance value was a pure white; the one with the lowest value, a deep black; the one with the middle value, a mid gray. The other squares were described as transition values from white to gray to black. In Experiment II, the modulus was referred to as a gray disk and each $O$ was given instructions concerning the difference between the whiteness and brightness dimensions. The $\mathrm{E}$ informed the $O$ that the whiteness dimension varied from white through gray to black while the brightness dimension varied from very bright to very $\mathrm{dim}$.

\section{Results and Discussion}

Experiment I. Log geometric means $(n=20)$ were determined for each contrast radio under the four levels of illumination. Whiteness functions (log geometric estimate vs illumination level in decibels) were plotted for each contrast ratio. These are represented in Fig. 2. It can be seen that the whiteness estimates for each of the six functions varied in accordance with the target's contrast ratio (i.e., targets under greater contrast were given lower estimates). Each point represents the average whiteness estimate for 10 Os. Log geometric means (n $=2$ ) were also determined for each $\mathrm{O}$ under the 24 experimental conditions. These estimates were then arranged into six whiteness functions describing the variation in whiteness estimates with changes in illumination for a constant contrast ratio (i.e., they indicate the tendency toward constancy for a given contrast ratio). The obtained functions (constancy function) were subjected to regression and trend analyses.

The constancy functions were fitted with straight lines using a dummy variable regression technique. This is an extension of the normal multiple linear regression model and has been described by Cohen (1968) as well as in texts on econometric analyses (i.e., Goldberger, 1964, pp. 224-231). In the present context, each $O$ was coded as a dummy variable.
Fig. 1. Stimulus configurations for Experiment I (reference in the upper left; test stimulus in the lower left) and Experiment IV (reference in the upper right; test stimulus in the lower right).
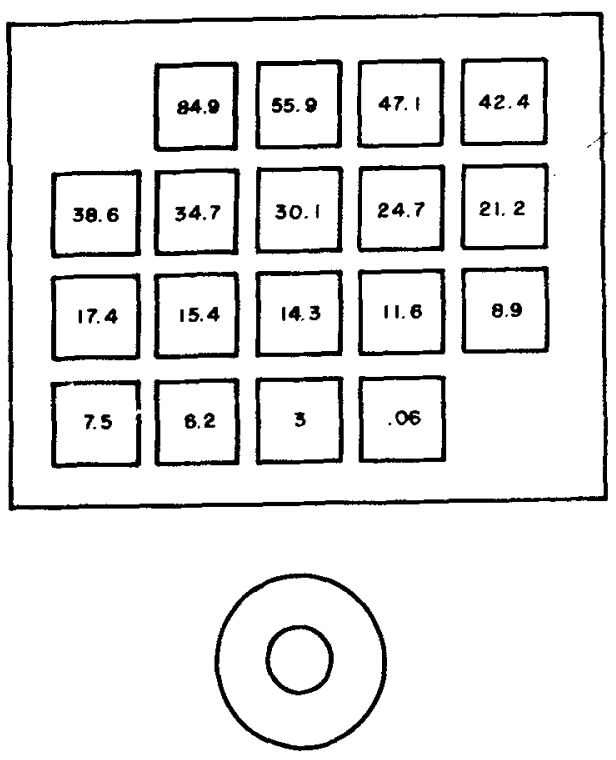

EXPERIMENT I
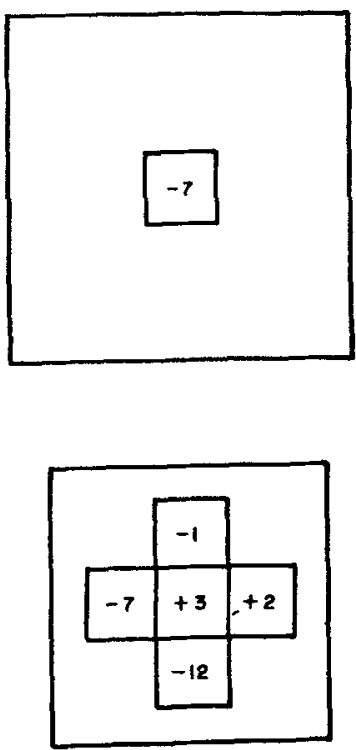

EXPERIMENT F 


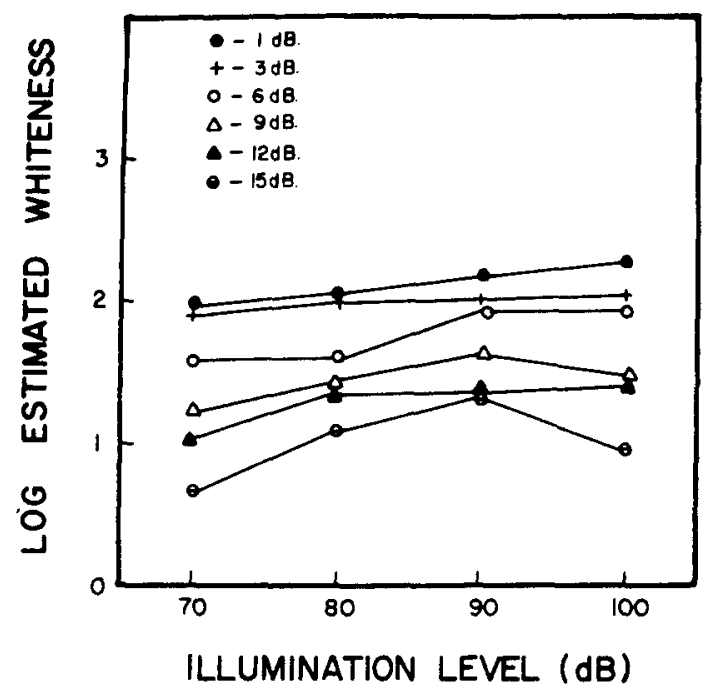

Fig. 2. Constancy functions obtained in Experiment I (log estimated whiteness as a function of illumination level for each contrast ratio).

Multiple linear regression functions were then fitted to the data using the actual independent variable (illumination level) and the dummy variables as independent variables and the estimates as the dependent variable. The derived constancy functions are shown in Fig. 3.

From the estimated functions, it was possible to obtain regression coefficients that reflected the change in the dependent variable with changes in the actual independent variable while the dummy variables were held constant. That is, the regression coefficients may be considered a measure of the function's slope in which the variability attributable to different Os has been partialed out. The obtained regression coefficients for the six constancy functions were $.12(-1 \mathrm{~dB}), .04(-3 \mathrm{~dB}), .15(-6 \mathrm{~dB}), .10(-9 \mathrm{~dB})$, $.12(-12 \mathrm{~dB})$, and $.04(-15 \mathrm{~dB})$. Student $t$ ratios were computed to determine whether the obtained coefficients differed significantly from values of 0 (Goldberger, 1964, p. 173). The computed t values for five of the constancy functions $(-1,-3,-6,-9$, and $-12 \mathrm{~dB}$ ) were significant, $\mathrm{p}<.05$.
A linear trend analysis was made on each of the six functions. The obtained $F$ values for four of the constancy functions $(-1,-6,-9$, and $-12 \mathrm{~dB})$ revealed a significant linear trend, $p<.05$. To measure the overall trend, the data were subjected to a 6 (contrast ratio) by 4 (illumination level) trend analysis with repeated measurements on both factors (Winer, 1962, pp. 367-368). Significant linear trends were found on the illumination level factor, $F(1,9)=14.42, \mathrm{p}<.01$, and the contrast ratio factor, $F(1,9)=31.7, \mathrm{p}<.01$. The Contrast Ratio by Illumination Level interaction, however, was not significant, $F(5,45)=1.68$, $\mathrm{p}>.05$.

Two major outcomes may be derived from these analyses. First, the individual regression functions, the trend analyses on the individual functions, and the overall trend analyses indicated that the six obtained constancy functions departed significantly from zero, i.e., perfect constancy was never obtained. Second, the nonsignificant Contrast Ratio by Illumination Level interaction and the significant trend on the contrast ratio factor indicated that the constancy functions for each contrast ratio possessed the same profile, i.e., they were a series of parallel and vertically displaced functions.

Experiment II. The whiteness estimates for each function varied in accordance with the target's contrast ratio. Constancy functions were derived using the dummy variable regression technique and are shown in Fig. 3. The obtained regression coefficients for the six constancy functions were $.12(-1 \mathrm{~dB}), .11$ $(-3 \mathrm{~dB}), .11(-6 \mathrm{~dB}), .21(-9 \mathrm{~dB}), .21(-12 \mathrm{~dB}), .25$ $(-15 \mathrm{~dB})$. The computed $t$ values for the constancy functions were all significant, $p<.001$.

All constancy functions showed significant linear trends, $F(1,9)=10.56, p<.01$. When the data were subjected to an overall trend analysis (6 by 4 with repeated measurements), significant linear trends were found on the illumination level factor, $F(1,9)=$ $47.26, \mathrm{p}<.01$; the contrast ratio factor, $F(1,9)=$ $35.6, \mathrm{p}<.01$; and the Contrast Ratio by Illumination Level interaction, $F(5,45)=2.70, p<.05$. Linear comparison values were computed for each $\mathrm{O}$ at each contrast ratio (Winer, 1962, p. 133). These values

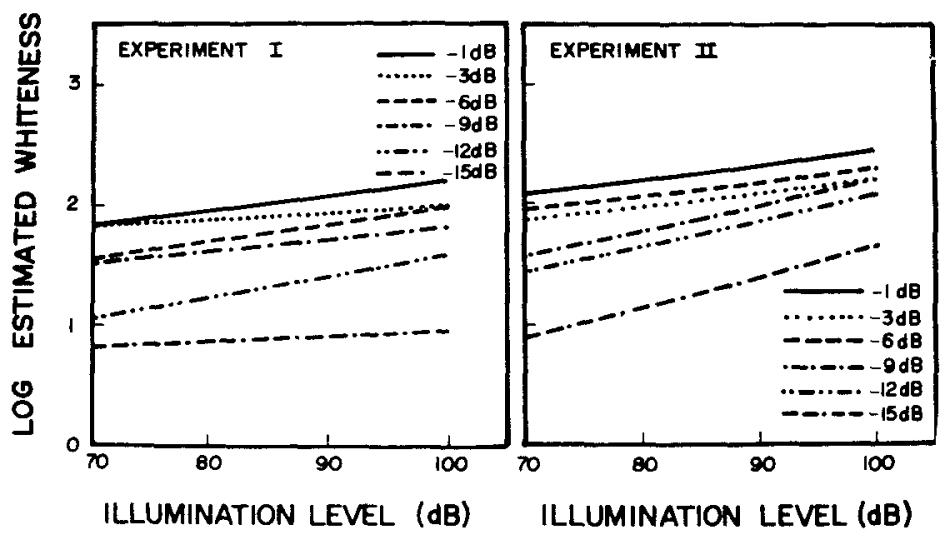

Fig. 3. Derived constancy functions for Experiments I (left panel) and II (right panel). 
were treated as the data in a trend analysis (linear comparison vs contrast ratio). The results indicated a significant linear trend, $F(1,9)=5.26, \mathrm{p}<.05$.

Several conclusions were suggested by these analyses. First, the constancy functions were linear with slopes that departed significantly from zero. Second, the six functions were a series of vertically displaced functions whose slopes increased as the contrast ratio decreased from -1 to $-15 \mathrm{~dB}$.

Experiments I and II. The constancy functions obtained in Experiment II differed in several ways from those found in Experiment I. First, regression and trend analyses revealed that all of the constancy functions derived from Experiment II were linear: This was not true of the functions from Experiment I. Second, the Contrast Ratio by lllumination Level interaction was found to be linear in Experiment II but not in Experiment I. Moreover, a further analysis of the linear comparison values in Experiment II indicated a linear trend. These analyses and an inspection of the obtained slopes suggested that the slopes of the constancy functions obtained in Experiment II increased as the contrast ratio changed from -1 to $-15 \mathrm{~dB}$.

The data from Experiments I and II were combined and subjected to a 2 (experiment) by 6 (contrast ratio) by 4 (illumination level) analysis of variance. Significance was obtained on the Experiment by Illumination Level interaction, $F(3,54)=3.47$, $p<.05$, and the Experiment by Contrast Ratio by Illumination Level interaction, $F(15,270)=2.61$, $\mathrm{p}<.05$. The significant Experiment by Illumination Level interaction and Fig. 3 indicate that whiteness estimates at each illumination level in Experiment II were higher than those in Experiment I, i.e., the targets in Experiment $I$ with the same luminance value as those in Experiment II were viewed as darker. Such results could be attributed to differences in the effectiveness of the surround that resulted from the use of surrounds with different areas. The significant Experiment by Contrast Ratio by Illumination Level interaction, as well as the slopes of the constancy functions, indicates that the average Whiteness by Illumination level slopes describing the data in Experiment II were steeper than those in Experiment I. That is, the tendency towards constancy was reduced in Experiment II.

It is also important to note that the slopes of the constancy functions obtained in Experiment II clustered into two groups. The slopes of the -1-, -3-, and $-6-\mathrm{dB}$ functions were approximately .11 ; those for the $-9-,-12-$, and $-15-\mathrm{dB}$ functions ranged from .21 to .25. The slopes of the latter were about twice the value of the former, as well as of those found in Experiment 1. In fact, they were close to the value of the slope of the standard brightness function (.33).

\section{EXPERIMENT III}

This experiment was designed to investigate the tendency towards brightness constancy when the modulus is viewed in the aperture mode and Os are instructed to adopt a brightness criterion.

\section{Method}

The test stimulus pattern, the contrast ratios, and illumination levels were the same as those used in Experiment I. The modulus was a disk of $1 \mathrm{deg}$ of visual angle, and appeared as a member of a series of disks located at a distance of $76.2 \mathrm{~cm}$ to the left of the $O$. Each disk in the series was formed from apertures of $1 \mathrm{deg}$ of visual angle in a piece of black construction paper. Each aperture was covered with Wratten No. 96 neutral density filters, mounted on a flashed opal plate, and transilluminated by several incandescent lamps. Since light only passed through the 1-deg apertures and the room was darkened, the disks appeared in the aperture mode. The luminance of the brightest disk was $64 \mathrm{~mL}(88 \mathrm{~dB})$; the luminance of the modulus was $6.4 \mathrm{~mL}(78 \mathrm{~dB})$.

The Os were given instructions concerning the brightness dimension. The $E$ pointed to the disk in the reference series with the highest, lowest, and middle luminance value, and informed $O$ s that the one with the highest luminance value was very bright; the one with the lowest, very dim; the one with a middle value, moderately bright. The other disks were described as having transitional values, from bright to moderately bright to dim.

\section{Results and Discussion}

With one exception, the average ordinate value for each of the six functions varied in accordance with the target's contrast ratio. The $68-\mathrm{dB}$ disk (i.e., a disk with a $-12-\mathrm{dB}$ contrast ratio viewed under an $80-\mathrm{dB}$ illumination level) was judged as brighter than a 71- $\mathrm{dB}$ disk (a disk with a -9-dB contrast ratio under an $80-\mathrm{dB}$ illumination level). Constancy functions were derived using the dummy variable regression technique and are shown in Fig. 4. The obtained regression coefficients for the six constancy functions were $.17(-1 \mathrm{~dB}), .09(-3 \mathrm{~dB}), .22(-6 \mathrm{~dB}), .21(-9 \mathrm{~dB})$, $.18(-12 \mathrm{~dB})$, and $.24(-15 \mathrm{~dB})$. The computed t values for the constancy functions were all significant, $\mathrm{p}<.05$.

Trend analyses on the $-1-,-6-,-12-$, and $-15-\mathrm{dB}$ constancy functions revealed significant linear trends, $\mathrm{F}(1,9)=5.12, \mathrm{p}<.05$. An overall trend analysis $(6$ by 4 ) with repeated measurements revealed significant linear trends on the illumination level factor, $F(1,9)$ $=12.06, \mathrm{p}<.01$, and the contrast ratio factor, $F(1,9)=36.8, p<.01$. The Contrast Ratio by Illumination Level interaction, however, was not significant, $F(5,45)=1.68, p>.05$. Two conclusions may be derived from these analyses. First, the slope of the overall constancy function departed significantly from zero; second, the six constancy functions were a series of parallel and vertically displaced functions.

This study differed from Experiment $I$ in terms of the response criterion and the mode of appearance of the criterion (modulus). In this study, the Os were 


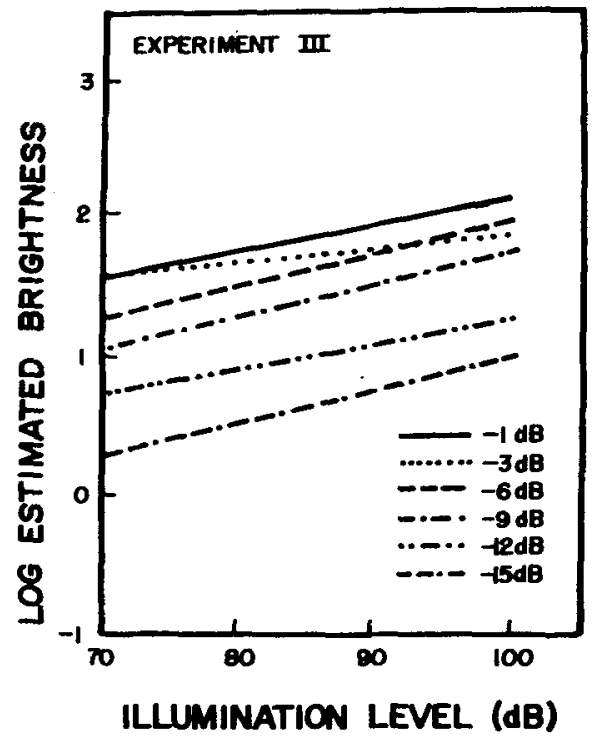

Fig. 4. Derived constancy functions for Experiment III.

instructed to give brightness estimates (brightness criterion) to targets viewed in the surface mode using as a modulus a target viewed in the aperture mode; in Experiment I, the Os were instructed to give whiteness estimates (whiteness criterion) to targets viewed in the surface mode, using as a modulus a target viewed in the surface mode. The data from Experiments I and III were combined and subjected to a 2 (experiment) by 6 (contrast ratio) by 4 (illumination level) analysis of variance. Significance was obtained on the experiment factor, $F(1,18)=$ $6.58, \mathrm{p}<.05$, and the Experiment by Contrast Ratio interaction, $F(5,90)=5.74, p<.01$. However, significance was not found on the Experiment by Illumination Level interaction, $F(3,54)=1.90$, $\mathrm{p}>.05$, or the Experiment by Contrast Ratio by Illumination Level interacţion, $F(15,270)=.923$, $\mathrm{p}>.05$. Thus, the whiteness estimates at each contrast level in Experiment $I$ differed in magnitude from the comparable brightness estimates in Experiment III, but the slopes in Experiment I did not differ from those in Experiment III.

The difference in response criterion did not seem to influence the slope of the constancy functions. In both experiments, the obtained constancy functions were a set of vertically displaced and parallel functions with shallow, but nonzero, slopes. It should be noted, however, that the test stimulus configuration was viewed in the surface mode and that it may have been difficult for the Os to respond to brightness changes when the whiteness of the test stimulus remained approximately constant. Greater differences between experiments could have been obtained if Os had been given extensive training on the response criterion.

\section{EXPERIMENT IV ${ }^{3}$}

This experiment was designed to evaluate the tendency towards constancy when Os were instructed to adopt a whiteness criterion and were shown a complex target configuration comparable to the one reported by Jameson and Hurvich (1961).

\section{Method}

A single test stimulus configutation composed of Wratten No. 96 neutral density filters mounted between flashed opal and clear glass plates appeared at a distance of $76.2 \mathrm{~cm}$ in the O's frontoparallel plane. It consisted of five squares (targets) arranged in a cross-shaped configuration. Each target subtended a visual angle of $1 \mathrm{deg}$. The cross was centered on a larger field (test background), which subtended a visual angle of $4.8 \mathrm{deg}$. The luminance of each component of the cross maintained a constant ratio to the luminance of the test background. The configuration and the specific contrast ratio in decibels for each target are indicated in Fig. 1.4 A positive value indicates that the luminance of the target was always higher than the luminance of its background; a negative value indicates the reverse. The entire test stimulus configuration was viewed under four levels of illumination. The resulting luminance values for the background were $66,76,86$, and $96 \mathrm{~dB}$.

The reference stimulus (modulus) was a transilluminated square of $1 \mathrm{deg}$ of visual angle, and appeared at a distance of $76.2 \mathrm{~cm}$ to the left of the $O$. The modulus was centered on a larger field (the modulus background), which subtended a visual angle of $5.7 \mathrm{deg}$. The entire reference stimulus pattern was transilluminated by several incandescent lamps. Throughout the entire experiment, the modulus background was held constant at $88 \mathrm{~dB}$ while the modulus was held constant at $81 \mathrm{~dB}$.

At the beginning of the session, the $\mathrm{E}$ informed the $\mathrm{O}$ that the modulus was a gray square, somewhere between black and white, and that he was to call it 100 . With this as a reference value, the $O$ was instructed to assign to each component of the cross a number proportional to its whiteness.

\section{Results and Discussion}

Constancy functions were derived and are shown in Fig. 5. The obtained regression coefficients were .16 $(+3 \mathrm{~dB}), .09(+2 \mathrm{~dB}), .09(-1 \mathrm{~dB}), .10(-7 \mathrm{~dB})$, and $.18(-12 \mathrm{~dB})$. The computed $t$ values were all significant, $\mathrm{p}<.05$.

All constancy functions showed significant linear trends, $F(1,9)=10.56, p<.01$. An overall trend analysis ( 5 by 4 with repeated measurements) revealed significant linear trends on the illumination level factor, $F(1,9)=31.60, p<.01$, and the contrast ratio factor, $F(1,9)=44.34, p<.01$. The Contrast Ratio by Illumination Level interaction was not significant, $F(4,36)=1.91, p>.05$.

Two conclusions may be derived from an inspection of the obtained functions along with the above analyses. First, the constancy functions were linear, with slopes that departed significantly from zero. Second, the five constancy functions were a series of parallel and vertically displaced functions. The test stimulus pattern used in this study was designed to replicate the one used by Jameson and Hurvich (1961). It consisted of five targets whose luminance values were less than $(-1,-7$, and $-12 \mathrm{~dB})$ or greater 
than $(+3$ and $+2 \mathrm{~dB})$ the luminance of the surround. In the present study, as well as in that of Jameson and Hurvich, the obtained slope values were less than the slope of the standard brightness function (i.e., .33). Thus brightness or whiteness contrast existed even when the luminance of the surround was less than that of the test field.

Other investigators (Leibowitz et al, 1955; Kozaki, 1963), utilizing a simple rectangle-frame configuration, have found major slope changes between conditions in which the surround had a greater luminance than the test field and those in which it did not. Under the latter luminance conditions, the findings indicated that Os were making luminance matches. According to these results, the surround did not act as a contrast-inducing field when its luminance was less than that of the test field. In fact, Heinemann (1955) reported a slight enhancement of the matched luminance of the test field when surrounded by a field with a slightly lower luminance value.

The data obtained in the present study, however, were also inconsistent with some of the findings reported by Jameson and Hurvich. In their study, the slopes of the constancy functions systematically changed from positive values through zero to negative values as the contrast ratios changed from +3 to $-12 \mathrm{~dB}$; in the present study, all constancy functions were linear, with positive slopes that departed significantly from zero. There were some methodological differences between the two studies. In the Jameson and Hurvich study. Os made luminance matches of individual targets while the overall illumination varied over a $1.1 \log$ unit range (approximately from 68.8 to $79.9 \mathrm{~dB}$ ). The obtained matches were then converted into brightness values through a magnitude estimation procedure. Os in the present study made direct whiteness estimates of targets while the overall illumination varied over $3 \log$ units ( 66 to $96 \mathrm{~dB}$ ). Further, estimates were given after two $1-\mathrm{sec}$ exposures. Even though this is a methodological departure from most current studies of constancy, previous investigators (Katz, 1935; Henneman, 1935) have achieved good approximations to constancy with exposures of about $.75 \mathrm{sec}$. Under the present experimental conditions, limited exposure helped to control excessive variability in the O's adaptation level, which, as suggested by Stevens and Stevens (1963), could seriously distort whiteness estimates. It seems unlikely, however, that these inconsistent findings were solely the result of methodological differences. The discrepancy could be a function of the use of a larger $\mathbf{N}$ and the extensive statistical analyses of the data in the present study.

There also was a discrepancy between the data obtained in the present study and those of Flock and Noguchi (1970). The constancy functions in their study were linear and positive, but their slopes did appear to covary with contrast ratio. There were,

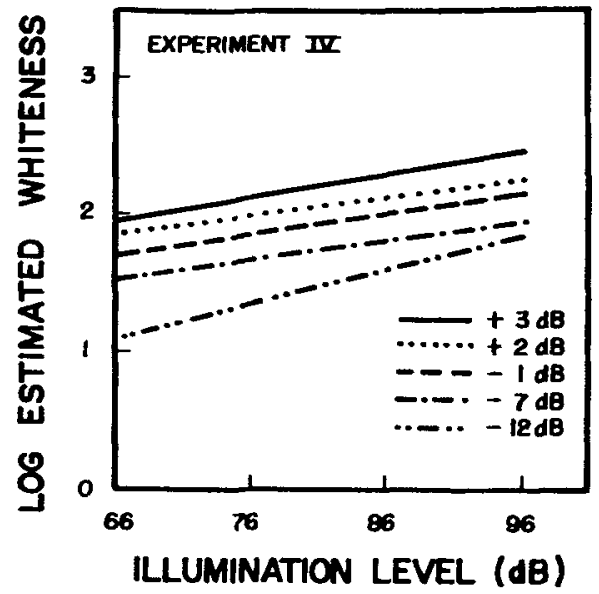

Fig. 5. Derived constancy functions for Experiment IV.

however, some methodological differences that could account for these differences. First, a different modulus was used for each test target. Second, Os were adapted to a constant level of luminance and were tested at one illumination level before going on to another. In the present study, the Os were adapted to the level of the modulus background, but both illumination level and contrast ratio were randomized. These apparent differences in outcome, however, could be nonsignificant, since there was no statistical confirmation of actual slope differences among the different functions in the Flock et al study.

\section{GENERAL DISCUSSION}

The results of Experiments I-IV suggest that a strong and uniform tendency towards constancy prevails over a variety of experimental conditions. These include a broad range of contrast ratios with the test stimulus either higher or lower in luminance than the surround, different stimulus configurations, and instructions to adopt either a whiteness or brightness criterion. Constancy, however, was lost when the area of the surround was reduced and the target and surround differed markedly in luminance (i.e., a contrast ratio of at least $-9 \mathrm{~dB}$ ).

Moreover, these findings do not confirm the existence of the relationship between contrast and constancy suggested by Jameson and Hurvich (1961). Decreasing the contrast ratio (i.e., increasing the magnitude of contrast) did not result in a progressive decrease in slope. The functions within Experiments I, III, and IV were vertically displaced and parallel; those within Experiment II were vertically displaced and increased in slope. This suggests two implications regarding slope changes and contrast ratios. First, when the area of the surround is larger than the target, decreasing the contrast ratio decreases the apparent whiteness (or brightness) but does not alter the tendency towards constancy. Second, when the 
area of the surround is reduced to that of the target, decreasing the contrast ratio decreases both the apparent whiteness and the tendency towards constancy.

\section{REFERENCES}

Conen, J. Multiple regression as a general data-analytic system. Psychological Bulletin, 1968, 70, 426-443.

FLOCK, H. R., \& NoGUCHI, K. An experimental test of Jameson and Hurvich's theory of brightness contrast. Perception \& Psychophysics, 1970, 8, 129-136.

Forgus, R. H. Perception. New York: McGraw-Hill, 1966.

Freeman, R. B. Contrast interpretation of brightness constancy. Psychological Bulletin, 1967, 67, 165-187.

Gelb, A.'Die Farbenkonstanz der Sehdinge. Handbuch der normalen und pathologischen Physiologie, 1929, 12, 594-678.

Goldberger, A. S. Econometric theory. New York: Wiley, 1964.

HAIMsON, B. R. The relationship of brightness contrast to brightness constancy. (Doctoral dissertation, Boston University) Ann Arbor, Mich: University Microfilms, 1970. No. 70-22, 392.

Heinemann, E. G. Simultaneous brightness induction as a function of inducing- and test-field luminances. Journal of Experimental Psychology, 1955, 50, 89-96.

HenNeman, R. H. A photometric study of the perception of object color. Archives of Psychology, 1935, No. 179, 5-89.

Hess, C., \& Pretori, H. Messende Untersuchungen über die Gesetzmassigkeit des simultanen Helligkeitskontrastes. Graefes Archiv für Ophthalmologie, 1894, 40, 1-24. Cited by L. M. Hurvich and D. Jameson, The perception of brightness and darkness. Boston: Allyn \& Bacon, 1966.

Horeman, H. W. Inductive brightness depression as influenced by configurational conditions. Vision Research, 1963, 3, 121-130.

Horeman, H. W. Relations between brightness and luminance under induction. Vision Research, 1965, 5, 331-340.

Jameson, D., \& Hurvich, L. Complexities of perceived brightness. Science, 1961, 133, 174-179.
KATZ, D. The world of color. (Trans. R. B. MacLeod and C. W. Fox) London: Kegan Paul, 1935.

Kozaki, A. A further study in the relationship between brightness constancy and contrast. Japanese Psychological Research, 1963. 5. 129-136.

LANDAUER, A. A., \& Rodger, R. S. Effect of "apparent" instructions on brightness judgments. Journal of Experimental Psychology, 1964, 68, 80-84.

Leibowitz, H., Myers, N. A., \& Chinetti, P. The role of simultaneous contrast in brightness constancy. Journal of Experimentäl Psychology, 1955, 50, 15-18.

MACLEOD, R. B. An experimental investigation of brightness constancy. Archives of Psychology, 1932, No.135, 5-102.

Stevens, J. C. Brightness inhibition re size of surround. Perception \& Psychophysics, 1967, 2, 189-192.

Stevens. J. C., \& Stevens, S. S. Brightness function: Effects of adaptation. Joumal of the Optical Society of America, 1963, 53, 375-385.

WALLACH, H. Brightness constancy and the nature of achromatic colors. Journal of Experimental Psychology, 1948, 38, 310-324.

WINER, B. J. Statistical principles in experimental design. New York: McGraw-Hill, 1962.

\section{NOTES}

1. $10 \times \log$ (disk luminance/annulus luminance).

2. Re: $10-10 \mathrm{~L}(70 \mathrm{~dB}$ is equivalent to $1 \mathrm{~mL}$ ).

3. Experiment IV was reported at the meeting of the Eastern Psychological Association, Atlantic City, April 1970.

4. The contrast ratio of the targets in the Jameson and Hurvich study was derived from information given in Freeman (1967). The obtained values were very close to the values in the present study.

(Received for publication February 6, 1974; revision received June 11,1974 .) 